\title{
Electrospinning Preparation of Timosaponin B-II-Loaded PLLA Nanofibers and Their Antitumor Recurrence Activities In Vivo
}

\author{
Zhonghua Huo, ${ }^{1}$ Yan Qiu, ${ }^{2}$ Zhuling Chu, ${ }^{1}$ Peng Yin, ${ }^{1}$ Wenquan Lu, ${ }^{3}$ \\ Yijia Yan, ${ }^{4} \mathrm{Na}$ Wan, ${ }^{4}$ and Zhilong Chen ${ }^{4}$ \\ ${ }^{1}$ Department of General Surgery, 454th Hospital of PLA, Najing 210002, China \\ ${ }^{2}$ Department of Pharmacy, 454th Hospital of PLA, Najing 210002, China \\ ${ }^{3}$ Department of Pharmacy, Shanghai Changzheng Hospital, 415 Fengyang Road, Shanghai 200003, China \\ ${ }^{4}$ Department of Pharmaceutical Science \& Technology, College of Chemistry and Biology, Donghua University, \\ 2999 North Renmin Road, Shanghai 201620, China
}

Correspondence should be addressed to Wenquan Lu; luwenquan@126.com and Zhilong Chen; chenzhilongdhu@gmail.com

Received 30 June 2014; Revised 11 September 2014; Accepted 15 September 2014

Academic Editor: Fujuan Liu

Copyright (c) 2015 Zhonghua Huo et al. This is an open access article distributed under the Creative Commons Attribution License, which permits unrestricted use, distribution, and reproduction in any medium, provided the original work is properly cited.

\begin{abstract}
Poly(L-lactic)-acid (PLLA) as a drug carrier and a water-soluble drug timosaponin B-II (TB-II) as a model drug were selected to prepare drug-loaded nanofibers by electrospinning. The average diameters of pure PLLA nanofibers and TB-II-loaded nanofibers were $212.5 \pm 68.5,219.7 \pm 57.8,232.8 \pm 66.9$, and $232.9 \pm 97.7 \mathrm{~nm}$, respectively. DSC and XRD results demonstrated that TB-II was well incorporated into the nanofibers in an amorphous state. FI-TR spectroscopy indicated that TB-II had good compatibility with PLLA. In vitro release studies showed that TB-II was rapidly released from the nanofibers within $6 \mathrm{~h}$, followed by a gradual release for long time. In vivo biosafety test revealed no noticeable toxicity of these TB-II nanofibers. The TB-II released from the nanofibers had obvious inhibition effect against human hepatocellular carcinoma SMMC 7721 cells both in vivo and in vitro. It was confirmed that the TB-II-loaded nanofibers were a sustained delivery system which could effectively inhibit the tumor growth and recurrence after surgery.
\end{abstract}

\section{Introduction}

Classically, surgery is the first-line treatment for most solid tumors [1]. However, it is difficult to completely remove the tumor through surgery [2]. The residual cancer cells remaining at or near the resection margins or site of initial treatment always initiate a local recurrence of tumors $[3,4]$. Thus, chemotherapy has been applied widely as an adjuvant procedure followed by surgery.

In recent years, substances derived from medical plants, such as paclitaxel, camptothecin, and vincristine, have been confirmed to be useful as chemotherapy drugs [5]. Anemarrhenae rhizoma (Zhimu in Chinese), the dried rhizome of A. asphodeloides Bunge (A. asphodeloides, Faro. Liliaceae), is a well-known traditional Chinese medicinal herb which has long been included in Chinese traditional medical recipes for treatment of inflammation, fever, and diabetes $[6,7]$.
Timosaponin B-II (TB-II) (Figure 1) is a typical furostanol saponin isolated from the rhizome of $A$. asphodeloides [8]. It has been reported that TB-II has remarkable inhibiting effects on superoxide generation, inflammatory reaction, and platelet aggregation potential $[9,10]$. Recent research has shown that TB-II will be converted to Timosaponin A-III (TA-III) by glycosidase treatment in vivo, which can induce apoptosis in various cancer cell lines [11].

Since chemotherapy drugs exhibit low selectivity, they destroy both tumor cells and normal cells. A locoregional drug administration need to be studied to avoid the systemic toxicity associated with chemotherapy drugs and maintain their therapeutic concentrations in the local region of tumors [8]. Electrospinning has been proven to be a great fabrication method for drug delivery systems due to the large selection of possible synthetic and natural, biodegradable or nondegradable polymers, and large surface-to-volume ratio [12, 13]. 


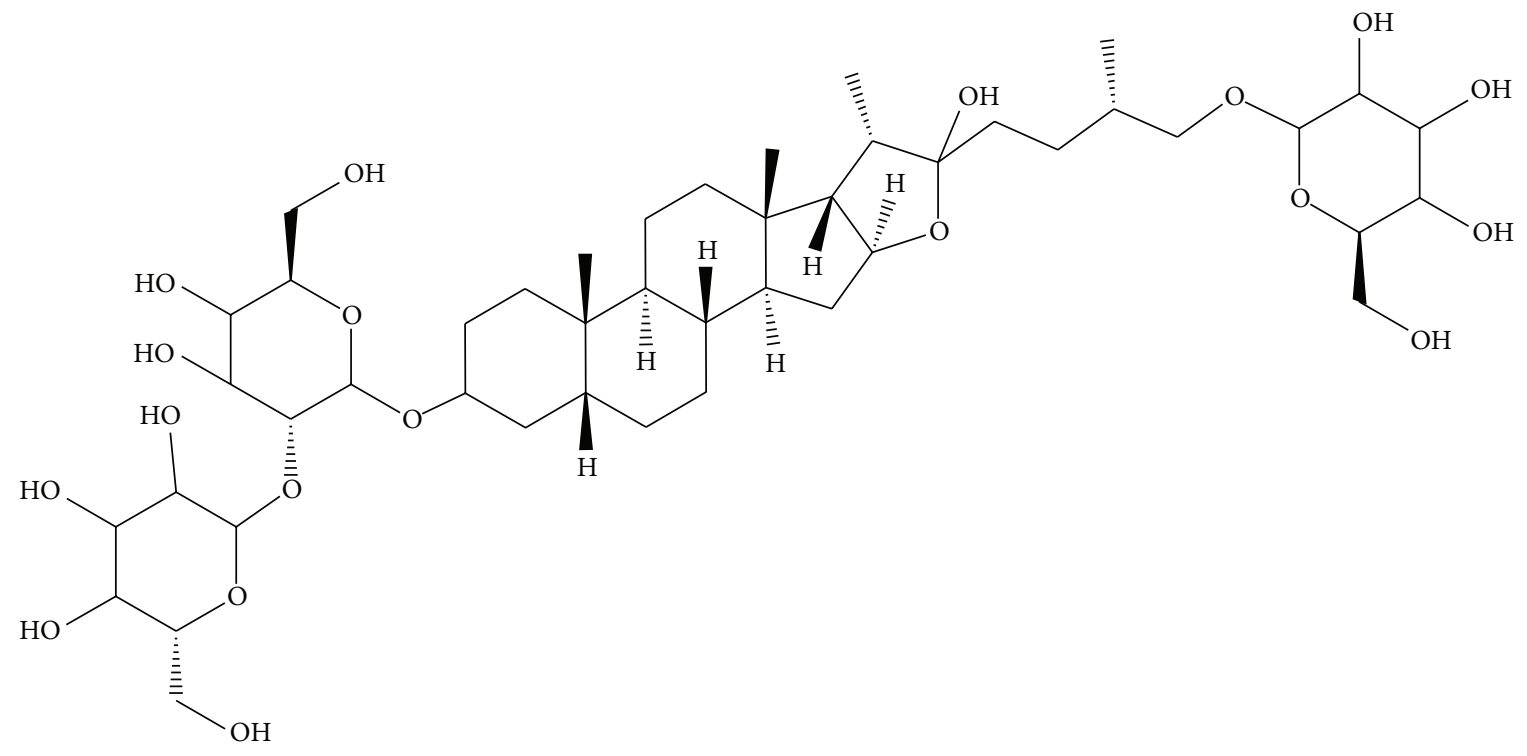

Figure 1: Chemical structures of TB-II.

The drug-loaded fibers prepared by electrospinning can be implanted intratumorally, adjacent to the cancerous tissue or at the surgical resection margins for cancer chemotherapy of solid tumors $[14,15]$. For preventing the recurrence of tumor, the drug-loaded fibers can be attached to the surgical site following removal of the tumor. As the drugs were moderately released from the sustained-release carriers, relatively steady effective concentration can be achieved to inhibit tumor growth $[4,14]$.

In this study, the poly(L-lactic)-acid (PLLA), a promising material with good biocompatibility, biodegradability, and nontoxic property, was selected as a drug carrier. TBII-loaded PLLA nanofibers were successfully prepared by electrospinning and their antitumor activities were evaluated both in vitro and in vivo.

\section{Materials and Methods}

2.1. Materials. TB-II with at least purity $95 \%$ was obtained from the Second Military Medical University. Poly(L-lactic) acid (PLLA), with an average molecular weight of 100,000, was purchased from Jinan Daigang Biomaterials company. RPMI 1640 and DMED were purchased from Shanghai Gino biological Ltd. All other chemicals and reagents were of analytical grade and used without any purification.

2.2. Animals. BALB/c-nu rats, 5 weeks, were provided by Hanghai SLAC Laboratory Animal Company. All animals were housed individually with water and food available and quarantined for 7 days prior to initiation of the study. A $12 \mathrm{~h}$ light/12 h dark cycle (light was switched at 06:00) was used. Room temperature was ranged from $22^{\circ} \mathrm{C}$ to $27^{\circ} \mathrm{C}$ and the humidity was maintained between $30 \%$ and $70 \%$. All animal procedures were approved by the University Animal Care and Use Committee.
2.3. Cell Lines and Culture. Human hepatocellular carcinoma cell line SMMC-7721 was purchased from Chinese Academy of Sciences. The cells were cultured in RPMI 1640 medium, supplemented with $10 \%$ fetal calf serum, and incubated at $37^{\circ} \mathrm{C}$ in a humidified atmosphere containing $5 \% \mathrm{CO}_{2}$.

\subsection{Preparation of TB-II-Loaded PLLA Nanofibers by Electrospinning}

2.4.1. Preparation of Spinning Solutions. The concentration of PLLA in the spinning solution was fixed at $5 \%(\mathrm{w} / \mathrm{v})$ according to preexperiments about its filament-forming properties. TB-II was mixed with PLLA to achieve a sustained release, and then the mixture was dissolved in chloroform/acetone solution (2/1 in volume ratio) and stirred for at least $3 \mathrm{~h}$ at room temperature. The weight ratio of TB-II in spinning solution that ranged from 10 to 15 was studied.

2.4.2. Electrospinning Process. Spinning solution was loaded in a $5 \mathrm{~mL}$ syringe to which a stainless-steel blunt needle was attached. The outer diameter of blunt needle was $1.0 \mathrm{~mm}$, and the inner diameter was $0.7 \mathrm{~mm}$. The needle tip was connected to an electrode of the high voltage power supply (DW-P503-1ACDF, Tianjin Dongwen High Voltage Power Supply Co., Ltd. China), and an $18 \mathrm{kV}$ of electrical potential was applied. The flow rate of solutions was controlled at $1.0 \mathrm{mLh}^{-1}$ by the syringe pump (LSP01-1A, Hebei Baoding LongerPump Co., Ltd. China). Randomly nonwoven fibers were collected on a metal collector wrapped with aluminum foil which was kept at a distance of $20-22 \mathrm{~cm}$ from the needle tip. Formed fibers were dried initially for over $24 \mathrm{~h}$ at $25^{\circ} \mathrm{C}$ under vacuum to remove residual solvent. 
2.5. Characterization. The morphology of the nanofibers was observed with a scanning electron microscopy (SEM, JEOL JSM-5600LV, Japan) at a voltage of $15 \mathrm{kV}$. The fiber average diameter was determined by measuring 50 fibers selected randomly from each sample using software image. Differential scanning calorimetry (DSC) analyses were performed on a MDSC 2910 differential scanning calorimeter (TA Instruments Co., DE, USA) at a heating rate of $10^{\circ} \mathrm{C} / \mathrm{min}$. Xray diffraction (XRD) patterns were obtained using a $\mathrm{D} / \mathrm{max}$ 2550PC (Geigerflex, Rigaku, Japan) with monochromated $\mathrm{CuKa}$ radiation operated at $40 \mathrm{kV}$ and $300 \mathrm{~mA}$. Fourier transformed infrared spectroscopy (FT-IR) was conducted using a Nicolet-Nexus 670 FTIR spectrometer (Nicolet Instrument Corporation, WI, USA) over the scanning range $500-4000 \mathrm{~cm}^{-1}$ with a resolution of $2 \mathrm{~cm}^{-1}$.

2.6. Release of TB-II from the Nanofibers In Vitro. To evaluate the in vitro release of TB-II, $10 \mathrm{mg}$ of drug-loaded PLLA nanofibers was incubated in $50 \mathrm{~mL}$ of phosphate buffer solution (PBS, $\mathrm{pH}=7.4$ ) at $37^{\circ} \mathrm{C} .2 \mathrm{~mL}$ of the sample solution was collected and diluted using fresh PBS at each time point. The amount of TB-II was monitored using a UV-vis spectrophotometer (7600CRT, Jinghua Instruments, China) at the wavelength of $280 \mathrm{~nm}$. All the measurements were carried out in triplicate and the average values were shown in this study.

2.7. Biosafety Test In Vivo. The nanofibers were cut to $1 \mathrm{~cm} \times$ $1 \mathrm{~cm}$ pieces and sterilized by exposure to UV light for $24 \mathrm{~h}$ before implantation. BALB/c-nu rats (weighing $20 \mathrm{~g}$ ) were divided randomly into five groups $(n=3)$ as follows:

Groups 1-3: rats implanted with nanofibers containing different concentrations of TB-II;

Group 4: rats implanted with pure PLLA nanofibers;

Group 5: rats with no treatment (control group).

Animals were anesthetized with ethyl ether, and then nanofibers were implanted into subcutaneous sites in the dorsal thoracic region of the rats. Body weight and survival rate of rats were evaluated every day. The weight growth rate was calculated by the use of the following equation:

$$
\text { Weight growth rate }(\%)=\frac{\left(W_{d}-W_{0}\right)}{W_{0}} \times 100 \% \text {, }
$$

where $W_{d}$ and $W_{0}$ are the average weights of rats at day $t$ and day 0 after implantation, respectively.

2.8. Antitumor Activities In Vitro. In vitro antitumor activities of the nanofibers were determined by MTT assay. Briefly, the nanofibers with different concentrations of TB-II were fixed on bottom of the wells of a 48 -well plate, and then $5 \times 10^{4}$ SMMC 7721 cells were seeded on the membranes and incubated for $24 \mathrm{~h}, 48 \mathrm{~h}$, and $72 \mathrm{~h}$, respectively [16]. $40 \mu \mathrm{L}$ MTT $(5 \mathrm{mg} / \mathrm{mL})$ reagent was added to each well and incubated at $37^{\circ} \mathrm{C}$ for $4 \mathrm{~h}$. At the end of the incubation, the medium was removed and the formazan complex was solubilized with $300 \mu \mathrm{L}$ DMSO. Absorbance of the complex was measured with a microplate reader (Bio-Rad, California, USA) at a wavelength of $492 \mathrm{~nm}$ and cell viability was calculated.

2.9. Antitumor Activities In Vivo. The tumor model was established by subcutaneous injection of $5 \times 10^{6}$ SMMC 7721 cells in the left axilla of female BALB/c-nu rats (weighing $20 \mathrm{~g}$ ). When tumors reached approximately $1 \mathrm{~cm}$ in diameter (between 21 and 28 days after tumor injection), rats were randomized into four groups $(n=3)$ and the antitumor recurrence activity was determined after overlay of TB-IIloaded PLLA nanofibers on tumors:

Group 1: rats had $60 \%$ partial tumor resection and were treated with TB-II-loaded PLLA nanofibers;

Group 2: rats had complete tumor resection and were treated with TB-II-loaded PLLA nanofibers;

Group 3: rats had $60 \%$ partial tumor resection with no treatment;

Group 4: rats had complete tumor resection with no treatment.

Briefly, rats were anesthetized with ethyl ether and a small incision was made on the skin to expose the tumor. Partial or complete tumor resection was carried out. The TB-IIloaded PLLA nanofibers were laid over the resection site and then the wound was closed using subcutaneous suturing. Animals were cared and observed for tumor recurrence. The tumor volumes of animals were monitored every three days after treatment. The tumor volumes were calculated using the following formula: $V=L W^{2} / 2$, where $L$ is the long diameter and $W$ is the shot diameter. The tumor growth inhibition rate (RTG) was calculated by the use of the following equation: RTG $(\%)=\left(V_{d}-V_{0}\right) / V_{0} \times 100 \%$, where $V_{d}$ and $V_{0}$ are the average volumes of tumors at day $t$ and day 0 after treatment, respectively.

2.10. Statistical Analysis. Data were presented as mean \pm standard deviation (S.D.). Student's $t$-test was used to measure differences using the Origin 9.0 software package. Statistical significance was taken as $P<0.05$.

\section{Results and Discussion}

3.1. Characterization of TB-II-Loaded PLLA Nanofibers. The morphology and diameter distributions of the fibers with various concentrations of TB-II were analyzed using SEM (Figure 2). It could be observed that all of the fibers were randomly oriented and had smooth surfaces, which indicated that TB-II was uniformly distributed in the fibers. The average diameters of the nanofibers with different TB-II concentrations were $219.7 \pm 57.8 \mathrm{~nm}, 232.8 \pm 66.9 \mathrm{~nm}$, and $232.9 \pm 97.7 \mathrm{~nm}$, respectively, when the diameter of pure PLLA fibers was about $212.5 \pm 68.5 \mathrm{~nm}$. The result indicated that the concentration of TB-II affected the diameter of the fibers slightly. 

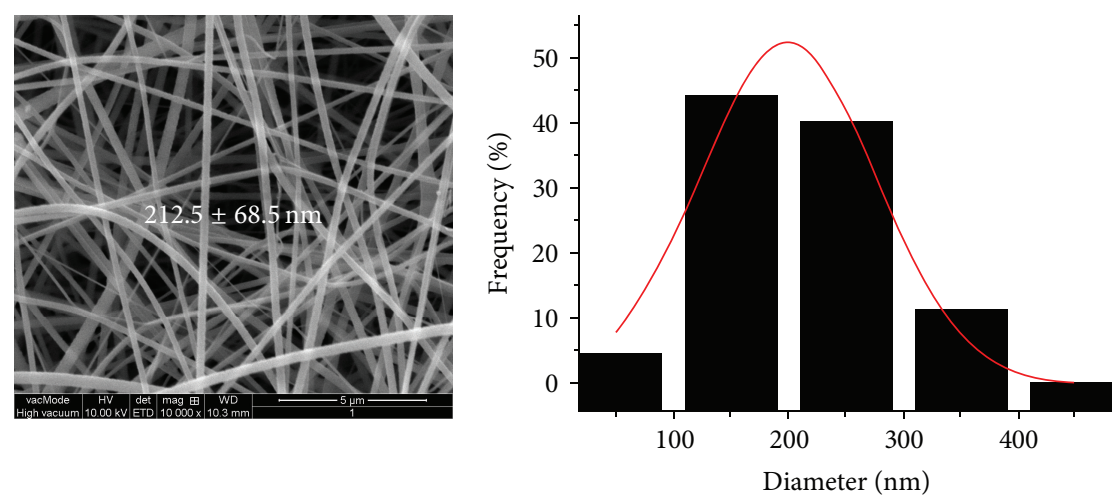

(a)
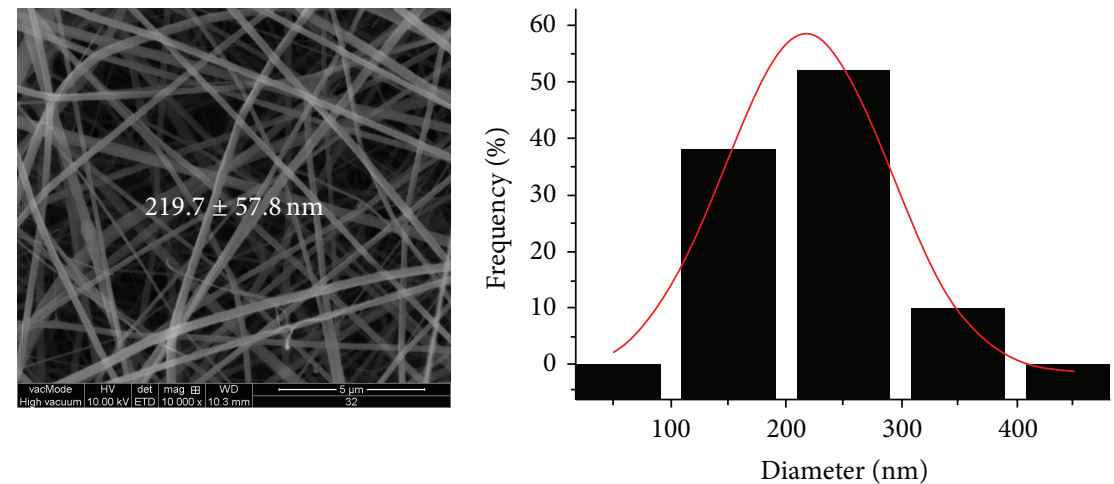

(b)
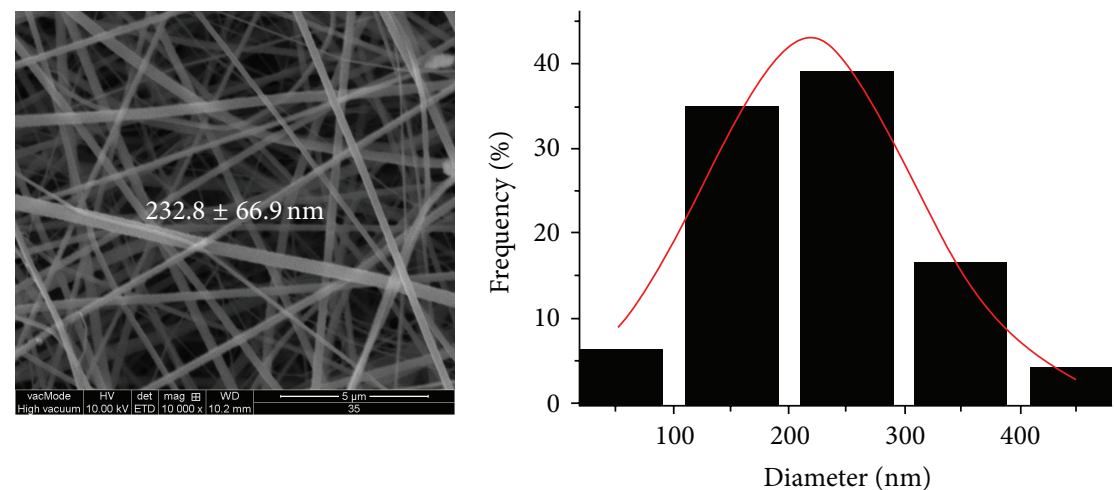

(c)
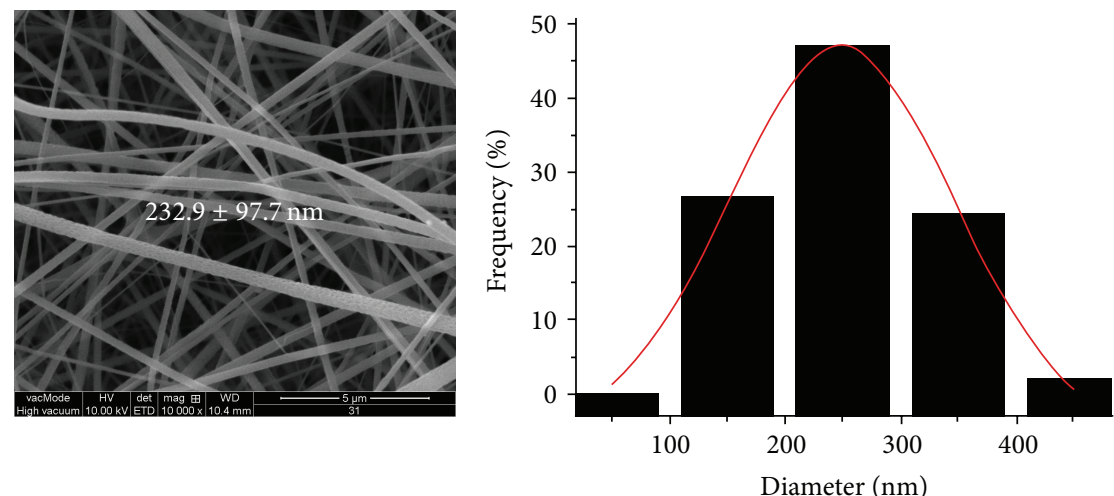

(d)

FIGURE 2: SEM images and diameter distributions of the pure PLLA nanofiber (a) and TB-II-loaded PLLA nanofibers with different drug concentration: (b) $10 \mathrm{wt} . \%$, (c) $12 \mathrm{wt} . \%$, and (d) $15 \mathrm{wt} . \%$. The scale bars are $5 \mu \mathrm{m}$. 


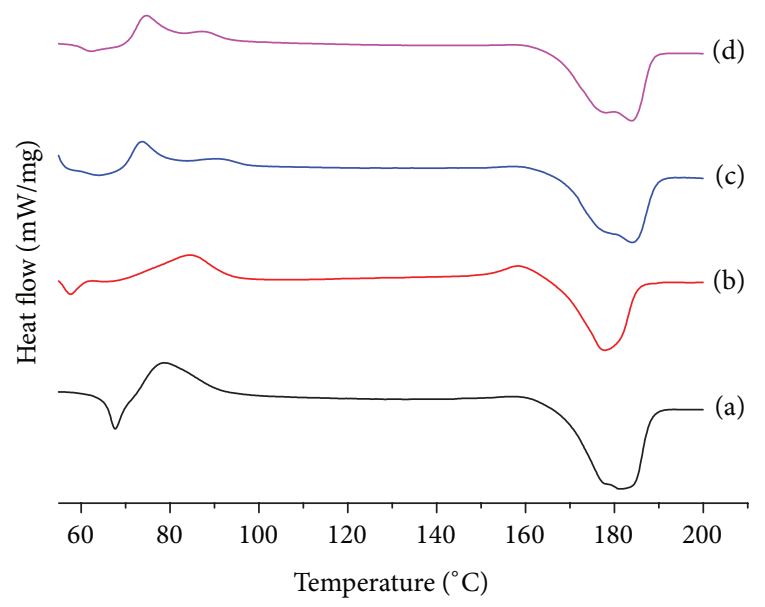

Figure 3: DSC curves of pure PLLA nanofiber (a) and TB-IIloaded nanofibers with different drug concentration: (b) $10 \mathrm{wt} . \%$, (c) 12 wt.\%, and (d) 15 wt.\%.

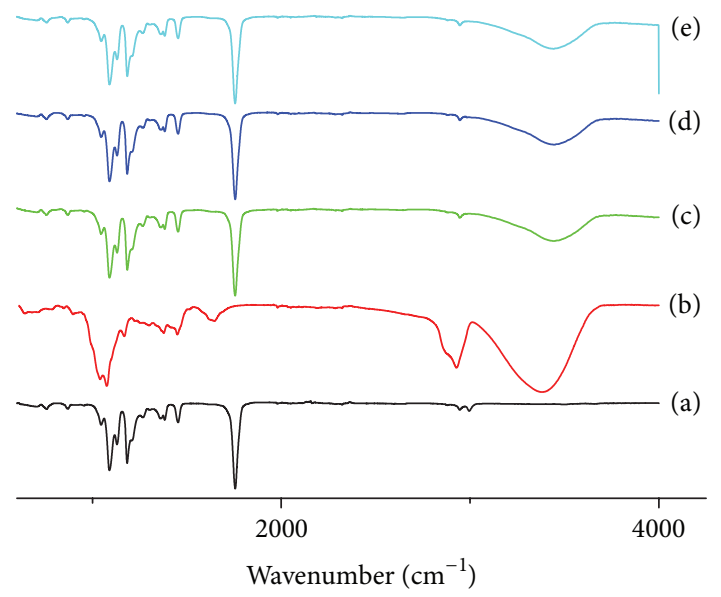

FIgURE 4: FT-IR spectra of TB-II (a), pure PLLA nanofiber (b), and TB-II-loaded nanofibers with different drug concentration: (c) 10 wt.\%, (d) 12 wt. $\%$, and (e) 15 wt.\%.

The DSC curves of PLLA nanofibers with and without different concentrations of TB-II were shown in Figure 3. The curve of pure PLLA nanofibers exhibited a single endothermic peak corresponding to melting at $181.2^{\circ} \mathrm{C}$. For the TB-II nanofibers, the DSC curves did not show any melting peaks of TB-II, suggesting that TB-II was not present as a crystalline material but had been converted into an amorphous state. The peak temperatures of the TB-II nanofibers were slightly shifted to $177.9,183.9$, and $183.7^{\circ} \mathrm{C}$ when the concentrations of TB-II increased to 10,12 , and $15 \mathrm{wt} . \%$, respectively. The result indicated that the concentrations of TB-II in the nanofibers had little effect on the thermal behavior.

The FT-IR spectra of pure PLLA nanofiber and TBII-loaded nanofibers were depicted in Figure 4. The TB-II samples were prepared by $\mathrm{KBr}$ pellet technique, and the nanofibers were scanned directly. The FI-TR spectrum of pure PLLA nanofibers showed characteristic peaks at $1756 \mathrm{~cm}^{-1}$ $(-\mathrm{C}=\mathrm{O}), 1090 \mathrm{~cm}^{-1}(-\mathrm{C}-\mathrm{O})$, and $1184 \mathrm{~cm}^{-1}(-\mathrm{C}-\mathrm{O})$. The spectrum of TB-II showed the dominant absorption peaks

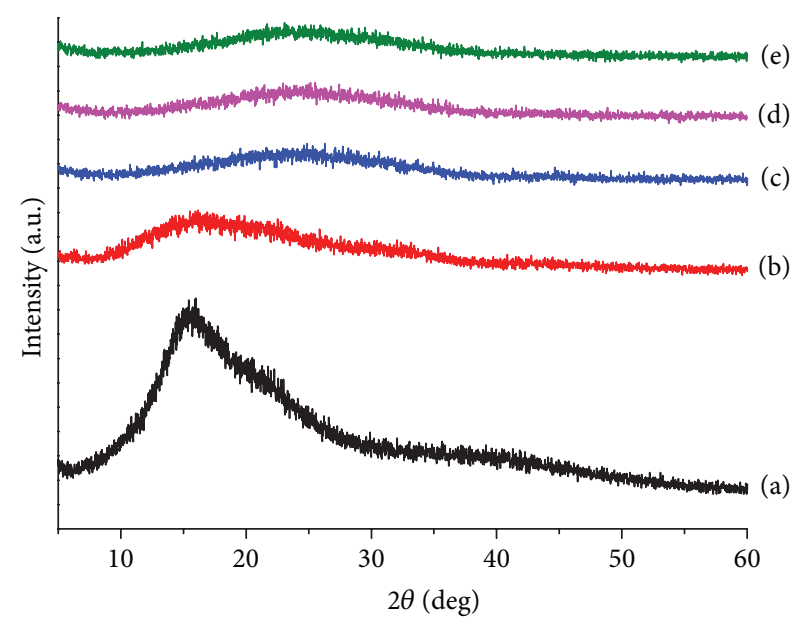

Figure 5: X-ray diffraction patterns of TB-II (a), pure PLLA nanofiber (b), and TB-II-loaded nanofibers with different drug concentration: (c) $10 \mathrm{wt} . \%$, (d) $12 \mathrm{wt} . \%$, and (e) $15 \mathrm{wt} . \%$.

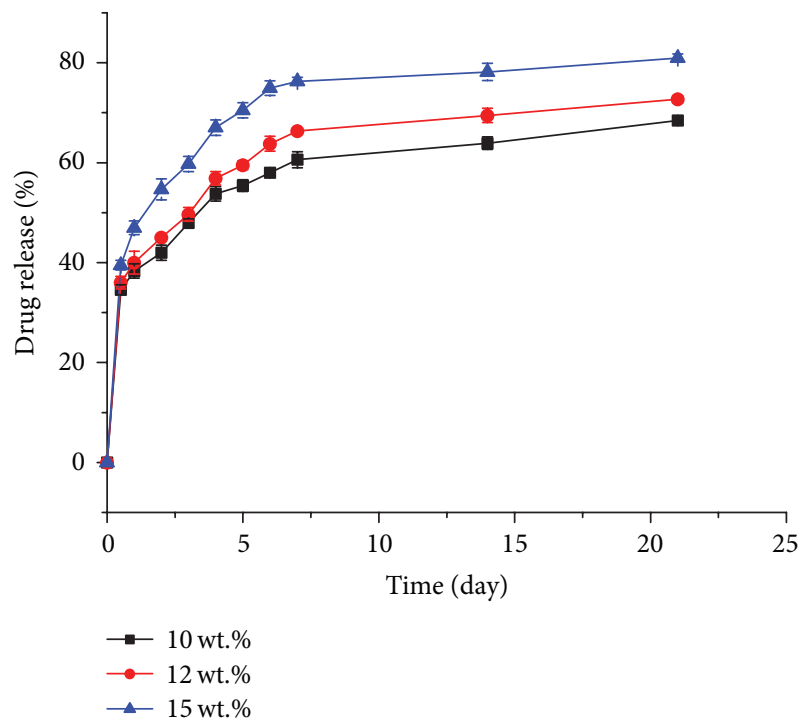

FIGURE 6: In vitro cumulative percentage of TB-II released from the nanofibers at various time points $(n=3)$.

at $3379 \mathrm{~cm}^{-1}(-\mathrm{O}-\mathrm{H}), 2928 \mathrm{~cm}^{-1}(-\mathrm{C}-\mathrm{H})$, and $1075 \mathrm{~cm}^{-1}$ (-C-O). The typical peaks corresponding to PLLA and TBII were both observed in the spectra of $10-15 \mathrm{wt} . \%$ TBII-loaded nanofibers, which indicated that TB-II had good compatibility with PLLA and was well incorporated into the nanofibers.

XRD patterns for the nanofibers were displayed in Figure 5. The pure PLLA nanofiber was amorphous material with a diffraction peak at $16.64^{\circ}$, when TB-II was a crystalline material with a strong peak at $15.94^{\circ}$. The XRD of all TB-II nanofibers showed that the characteristic peaks of PLLA and TB-II were both absent, suggesting that these two molecules were fully converted into an amorphous state. The result was further confirmed that TB-II was amorphously distributed in the nanofibers. 

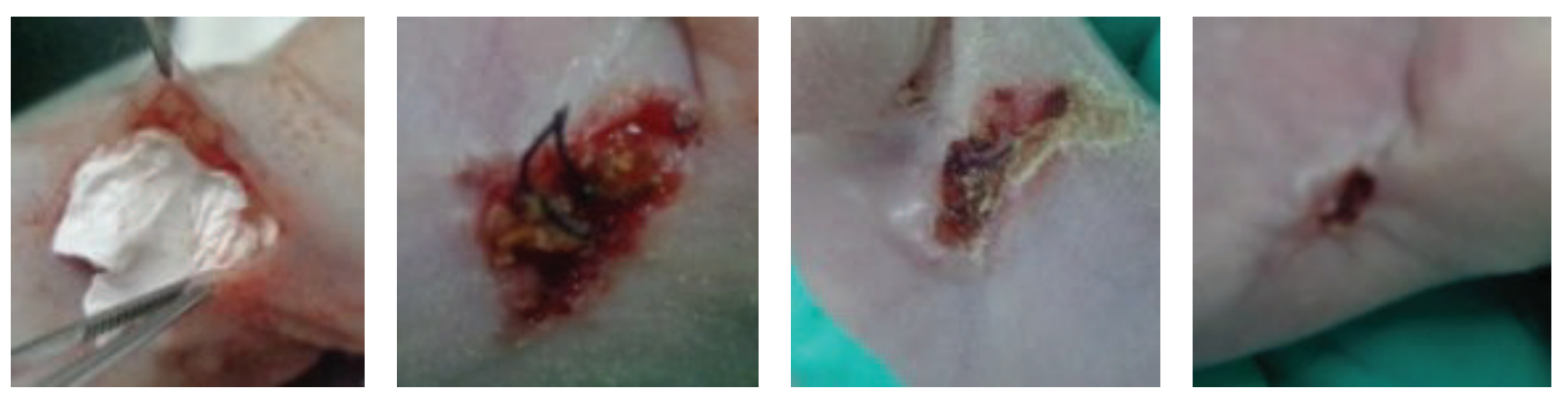

FIgURE 7: TB-II-loaded nanofibers implanted into subcutaneous sites in the dorsal thoracic region of the rats on days $0,7,14$, and 21.

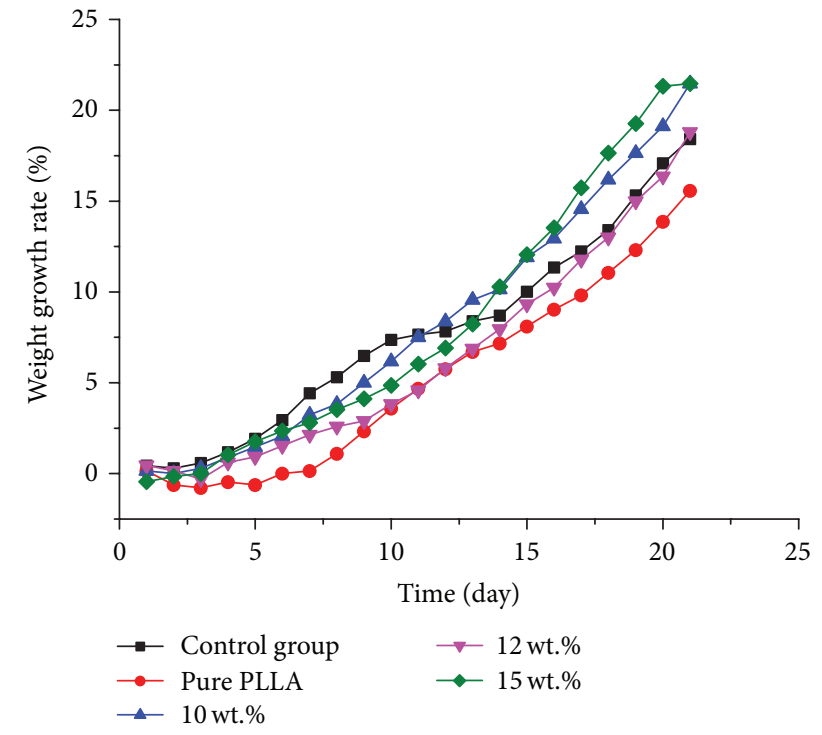

FIGURE 8: The weight growth rate of the rats after implantation by pure PLLA nanofibers and 10-15 wt.\% TB-II-loaded nanofibers $(n=3)$.

3.2. Release of TB-II from Nanofiber In Vitro. PLLA was selected as a drug carrier in this study as it is a relatively hydrophobic polymer because of the methyl group in its structure and therefore it can inherently slower biodegrade [16]. Drug release from the nanofibers with different concentrations of TB-II was shown in Figure 6. In all cases, TBII was rapidly released, followed by a gradual release. The amount of TB-II release within $6 \mathrm{~h}$ was $34.57,36$, and $39.5 \%$ corresponding to drug concentrations of 10,12 , and $15 \mathrm{wt} . \%$, respectively. This was probably due to the high concentration of the drug distributed on the electrospun nanofiber surface [17]. It could be observed that the release rate and maximum total amount of TB-II released from the nanofibers increased with the increasing of drug content. After 21 days, around $68.5,72.7$, and $81 \%$ of TB-II were released from the nanofibers with drug concentrations of 10,12 , and $15 \mathrm{wt} . \%$, respectively. The result indicated that the release of TB-II from the nanofibers might be continued for long time, suggesting that the TB-II nanofibers could be applied in the following in vitro and in vivo study.

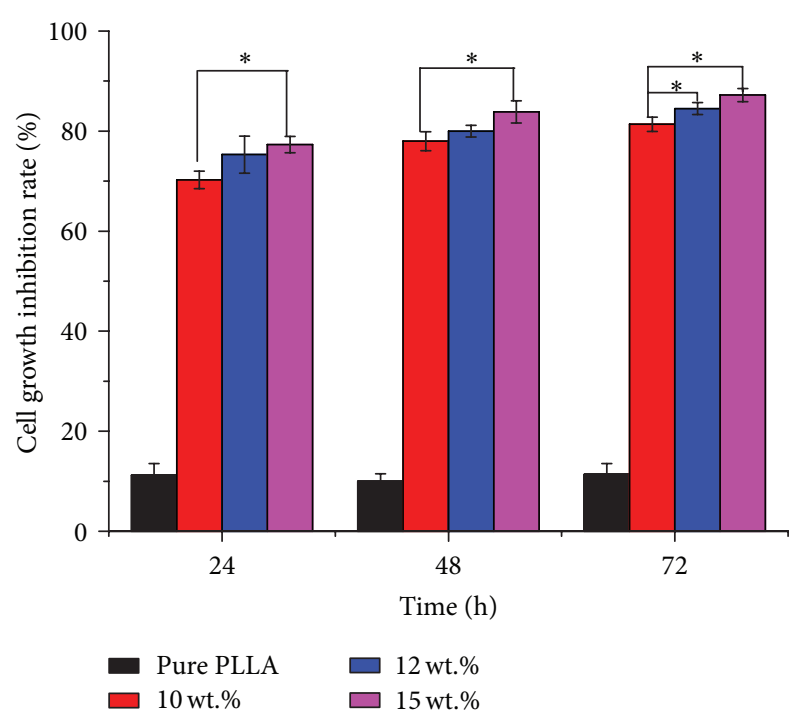

FIgURE 9: The growth inhibition rate of Human hepatocellular carcinoma SMMC 7721 cells on the pure PLLA nanofibers and 1015 wt.\% TB-II-loaded nanofibers $(n=3) .{ }^{*} P<0.05$.

3.3. Biosafety Test In Vivo. In order to investigate the toxicity of the TB-II nanofibers, the nanofibers were implanted into subcutaneous sites in the dorsal thoracic region of the rats (Figure 7). The body weight and survival rate were monitored every day. As shown in Figure 8, most rats experienced a slight weight loss within 3 days after implantation due to the pain-induced loss of appetite. However, the weight loss recovered in the following days, and there was no significant difference in the weight growth rate among the groups. All the animals survived within 21 days, indicating that there was no noticeable toxicity of the nanofibers with different concentrations of TB-II.

3.4. Antitumor Activities In Vitro. Liver cancer (LC) is the leading cause of cancer-related death worldwide [18]. Because of the high prevalence of the Hepatitis B virus, China has the highest mortality rate for LC [19-21]. Surgery is the only potentially curative treatment for LC [22]. However, tumor recurrence is still common after curative resection. Adjuvant treatment could be helpful in preventing tumor recurrence 

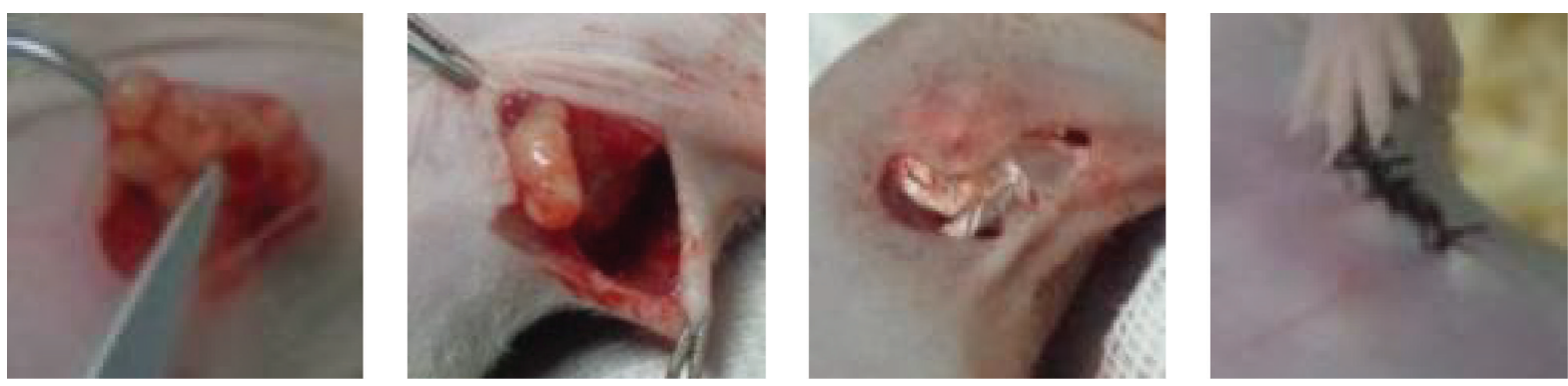

FIGURE 10: Rats had partial tumor resection and then TB-II-loaded nanofibers were laid over the tumor remnant.

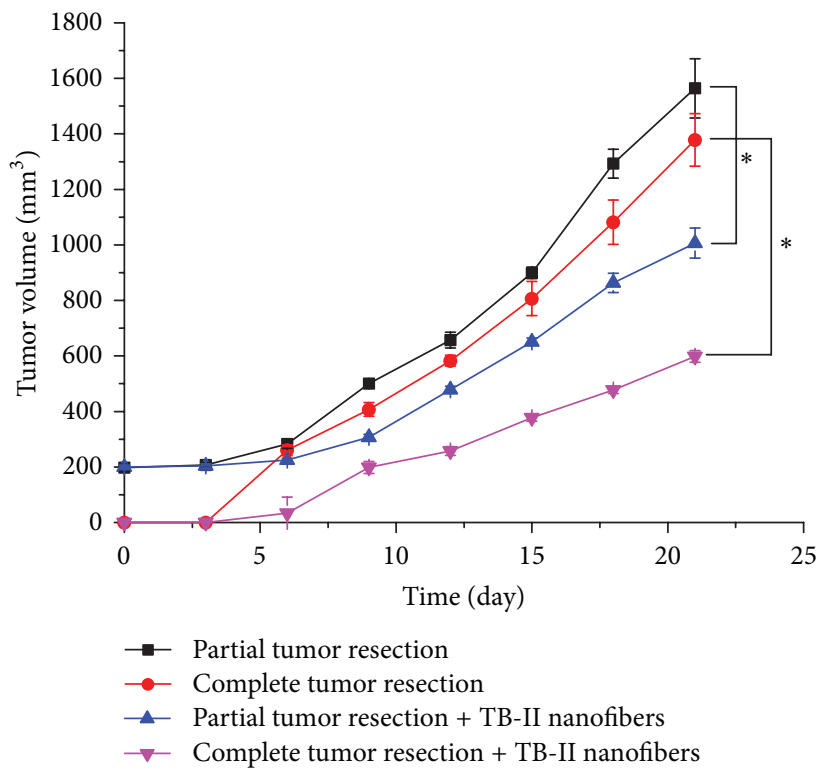

(a)

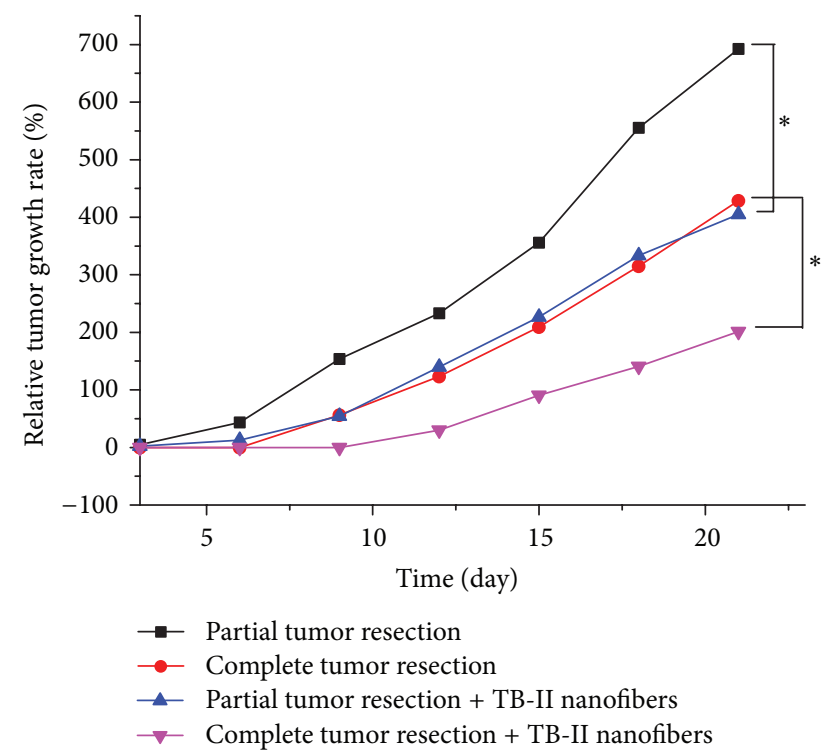

(b)

FIGURE 11: The tumor volume (a) and relative tumor growth rate (b) of rats after treatment with $15 \mathrm{wt} . \%$ TB-II-loaded PLLA nanofibers $(n=3)$. ${ }^{*} P<0.05$.

after partial surgical resection [23]. In this study, in vitro antitumor activities of TB-II-loaded nanofibers against human hepatocellular carcinoma SMMC 7721 cells were determined by MTT assay for 24,48 , and $72 \mathrm{~h}$. The results were shown in Figure 9. Compared to the pure PLLA nanofibers, all TB-II-loaded nanofibers showed very effective antitumor activity. A time-dependent and dose-dependent increase in the rate of cell growth inhibition rate could be observed, which indicated that TB-II could continuously be released in an active form from the nanofibers. Thus, the TB-II-loaded nanofibers were a sustained delivery system.

3.5. Antitumor Recurrence Activities In Vivo. According to the results of characterization and in vitro antitumor activities, PLLA nanofibers with $15 \mathrm{wt}$.\% TB-II were applied for the antitumor recurrence efficacy study in vivo. Animals had partial or complete tumor resection where the nanofibers were laid over, and then the wound was closed using subcutaneous suturing (Figure 10).

Figure 11 showed the tumor development after implantation of the TB-II-loaded nanofibers. In the cases of complete tumor resection, one of three rats treated with the TBII-loaded nanofibers presented macroscopic tumor (about $102 \mathrm{~mm}^{3}$ ) at day 9 when all three rats with no treatment presented macroscopic tumors (about $260 \pm 19 \mathrm{~mm}^{3}$ ) at day 6 . There was a significant difference both in the tumor volumes and RTG between control and treatment group. After 21 days, the tumor volumes of rats treated with the TB-II-loaded nanofibers reached around $598 \pm 20 \mathrm{~mm}^{3}$, while those of rats with no treatment reached around $1006 \pm 53 \mathrm{~mm}^{3}$. In the cases of partial tumor resection, tumor volumes of rats from the control and treatment group were $197 \pm 12$ and $199 \pm 8 \mathrm{~mm}^{3}$ at day 0, respectively. As shown in Figure 11, tumor volumes of rats treated with the TB-II-loaded nanofibers increased more slowly compared to those of rats with no treatment from the third day. The tumor volumes of rats treated with the TBII-loaded nanofibers reached around $1379 \pm 94 \mathrm{~mm}^{3}$ on day 21 , which was significantly smaller than those of rats with no treatment (about $1564 \pm 106 \mathrm{~mm}^{3}$ ).

The above results demonstrated the potential of the TB-II-loaded nanofibers as an implantable drug delivery system for liver cancer after surgery to effectively inhibit 
the tumor growth and recurrence. Therefore, the continuous inhibition of tumor growth after treatment with the TBII-loaded nanofibers confirmed further that TB-II could be continuously released from the nanofibers.

\section{Conclusions}

In this study, the TB-II-loaded nanofibers were prepared by electrospinning. The average diameter increased with the increase of TB-II content. DSC and XRD results demonstrated that TB-II was well incorporated into the nanofibers in an amorphous state. FI-TR spectroscopy indicated that TBII had good compatibility with PLLA. In vivo biosafety test revealed no noticeable toxicity of these TB-II nanofibers. In vitro release studies showed that TB-II was rapidly released from the nanofibers within $6 \mathrm{~h}$, followed by a gradual release for long time. The TB-II released from the nanofibers has obvious inhibition effect against human hepatocellular carcinoma SMMC 7721 cells both in vivo and in vitro. Thus, it was confirmed that the TB-II-loaded nanofibers were a sustained delivery system which could effectively inhibit the tumor growth and recurrence after surgery.

\section{Conflict of Interests}

The authors declare that there is no conflict of interests regarding the publication of this paper.

\section{Acknowledgments}

This work was supported by the Chinese National Natural Science Foundation (21372042, 81301878, and 81101298), Foundation of Shanghai government (13431900700, 13430722300, 13ZR1441000, and 13ZR1440900), the Medical Research Foundation of Nanjing Military Command (12mb020), Foundation of Donghua University (11D10501 and 12D10515), and Foundation of Yiwu Science and Technology Bureau (2011G1-15 and 2013623).

\section{References}

[1] D. R. Beil and L. M. Wein, "Sequencing surgery, radiotherapy and chemotherapy: insights from a mathematical analysis," Breast Cancer Research and Treatment, vol. 74, no. 3, pp. 279286, 2002.

[2] B. A. Pockaj and R. J. Gray, "Current surgery for breast cancer," Future Oncology, vol. 5, no. 4, pp. 465-479, 2009.

[3] P. Bouchard and J. Efron, "Management of recurrent rectal cancer," Annals of Surgical Oncology, vol. 17, no. 5, pp. 1343-1356, 2010.

[4] X. Luo, C. Xie, H. Wang, C. Liu, S. Yan, and X. Li, "Antitumor activities of emulsion electrospun fibers with core loading of hydroxycamptothecin via intratumoral implantation," International Journal of Pharmaceutics, vol. 425, no. 1-2, pp. 19-28, 2012.

[5] N. Wang, Y. Feng, M. Zhu, F.-M. Siu, K.-M. Ng, and C.-M. Che, "A novel mechanism of XIAP degradation induced by timosaponin AIII in hepatocellular carcinoma," Biochimica et Biophysica Acta-Molecular Cell Research, vol. 1833, no. 12, pp. 2890-2899, 2013.
[6] C. Guo, L. Li, X. Yang et al., "Protective effects of timosaponin BII on high glucose-induced apoptosis in human umbilical vein endothelial cells," Environmental Toxicology and Pharmacology, vol. 37, no. 1, pp. 37-44, 2014.

[7] T.-J. Li, Y. Qiu, P.-Y. Yang, Y.-C. Rui, and W.-S. Chen, “Timosaponin B-II improves memory and learning dysfunction induced by cerebral ischemia in rats," Neuroscience Letters, vol. 421, no. 2, pp. 147-151, 2007.

[8] S. Cheng, Y. Du, B. Ma, and D. Tan, "Total synthesis of a furostan saponin, timosaponin BII," Organic and Biomolecular Chemistry, vol. 7, no. 15, pp. 3112-3118, 2009.

[9] F. Cai, L. Sun, S. Gao, Y. Yang, Q. Yang, and W. Chen, "A rapid and sensitive liquid chromatography-tandem mass spectrometric method for the determination of timosaponin B-II in blood plasma and a study of the pharmacokinetics of saponin in the rat," Journal of Pharmaceutical and Biomedical Analysis, vol. 48, no. 5, pp. 1411-1416, 2008.

[10] Z. Liu, X. Dong, X. Ding et al., "Comparative pharmacokinetics of timosaponin B-II and timosaponin A-III after oral administration of Zhimu-Baihe herb-pair, Zhimu extract, free timosaponin B-II and free timosaponin A-III to rats," Journal of Chromatography B, vol. 926, pp. 28-35, 2013.

[11] F. W. King, S. Fong, C. Griffin et al., "Timosaponin AIII is preferentially cytotoxic to tumor cells through inhibition of mTOR and induction of ER stress," PLoS ONE, vol. 4, no. 9, Article ID e7283, 2009.

[12] A. Rogina, "Electrospinning process: versatile preparation method for biodegradable and natural polymers and biocomposite systems applied in tissue engineering and drug delivery," Applied Surface Science, vol. 296, pp. 221-230, 2014.

[13] T. J. Sill and H. A. von Recum, "Electrospinning: applications in drug delivery and tissue engineering," Biomaterials, vol. 29, no. 13, pp. 1989-2006, 2008.

[14] X. Luo, G. Xu, H. Song et al., "Promoted antitumor activities of acid-labile electrospun fibers loaded with hydroxycamptothecin via intratumoral implantation," European Journal of Pharmaceutics and Biopharmaceutics, vol. 82, no. 3, pp. 545-553, 2012.

[15] B. D. Weinberg, E. Blanco, and J. Gao, "Polymer implants for intratumoral drug delivery and cancer therapy," Journal of Pharmaceutical Sciences, vol. 97, no. 5, pp. 1681-1702, 2008.

[16] P. Chen, Q.-S. Wu, Y.-P. Ding, M. Chu, Z.-M. Huang, and W. $\mathrm{Hu}$, "A controlled release system of titanocene dichloride by electrospun fiber and its antitumor activity in vitro," European Journal of Pharmaceutics and Biopharmaceutics, vol. 76, no. 3, pp. 413-420, 2010.

[17] S. Huang, X. Kang, Z. Cheng, P. Ma, Y. Jia, and J. Lin, "Electrospinning preparation and drug delivery properties of $\mathrm{Eu}^{3+} / \mathrm{Tb}^{3+}$ doped mesoporous bioactive glass nanofibers," Journal of Colloid and Interface Science, vol. 387, pp. 285-291, 2012.

[18] Y.-H. Liao, C.-C. Lin, T.-C. Li, and J.-G. Lin, "Utilization pattern of traditional Chinese medicine for liver cancer patients in Taiwan," BMC Complementary and Alternative Medicine, vol. 12, article 146, 2012.

[19] A. Jemal, R. Siegel, E. Ward et al., “Cancer statistics, 2006," CA: A Cancer Journal for Clinicians, vol. 56, no. 2, pp. 106-130, 2006.

[20] B. H. Yang, J. L. Xia, L. W. Huang et al., "Changes of clinical aspect of PLC in China during the past 30 yearsdcontrol study for 3, 250 cases with PLC," National Medical Journal of China, vol. 83, pp. 1053-1057, 2003.

[21] P. Wang, Z. Q. Meng, Z. Chen et al., "Diagnostic value and complications of fine needle aspiration for primary liver cancer 
and its influence on the treatment outcome-a study based on 3011 patients in China," European Journal of Surgical Oncology, vol. 34, no. 5, pp. 541-546, 2008.

[22] M. A. Canosa, S. P. Fernández, J. Q. Fandiño et al., "Surgical treatment of liver cancer: experience of the A Coruña UHC (Spain)," Cirugía Española, vol. 89, no. 4, pp. 223-229, 2011.

[23] B. Aussilhou, Y. Panis, A. Alves, C. Nicco, and D. Klatzmann, "Tumor recurrence after partial hepatectomy for liver metastases in rats: prevention by in vivo injection of irradiated cancer cells expressing GMCSF and IL-12," Journal of Surgical Research, vol. 149, no. 2, pp. 184-191, 2008. 

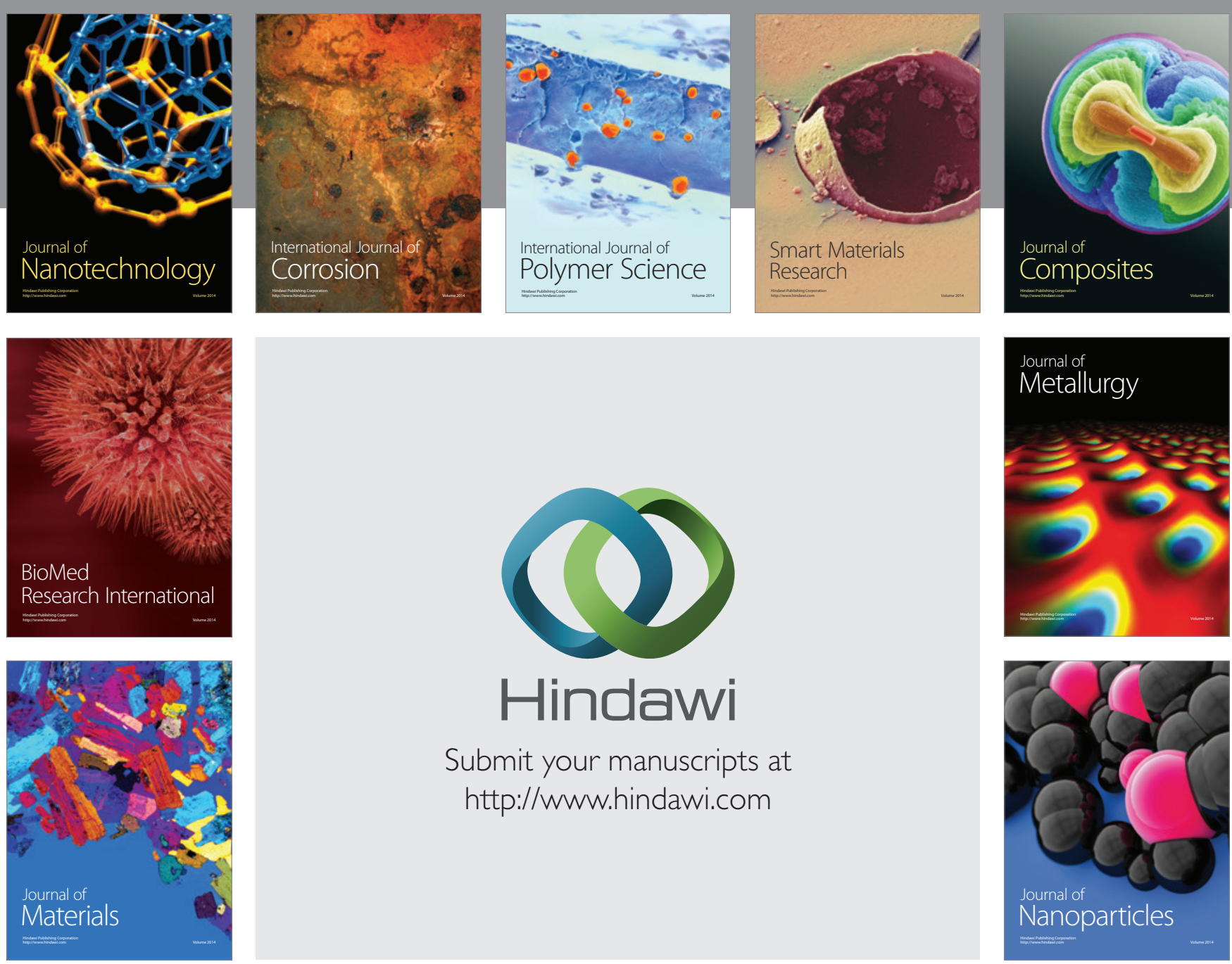

Submit your manuscripts at http://www.hindawi.com
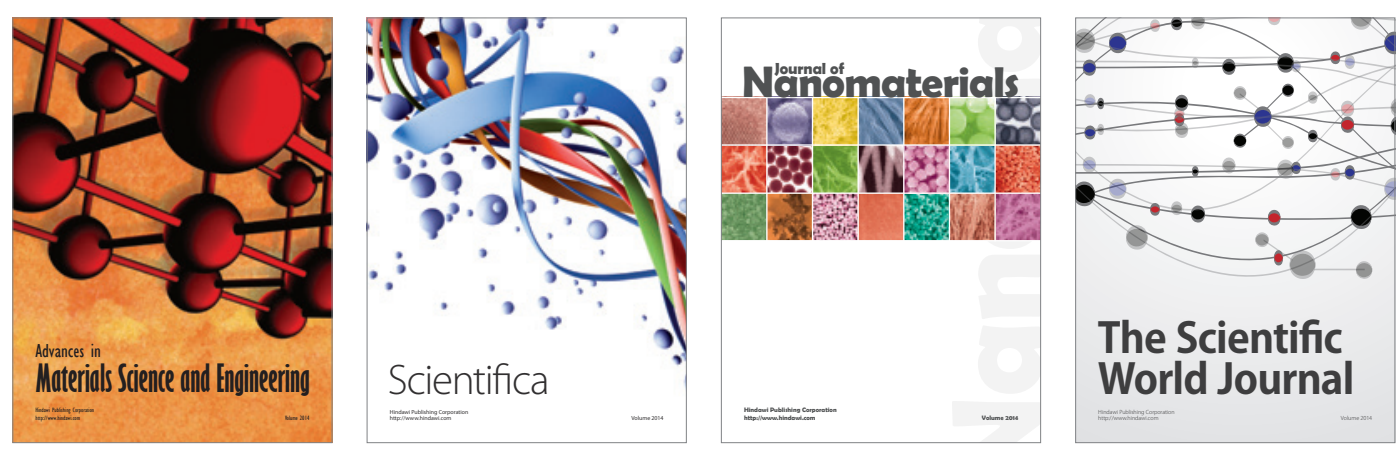

\section{The Scientific World Journal}
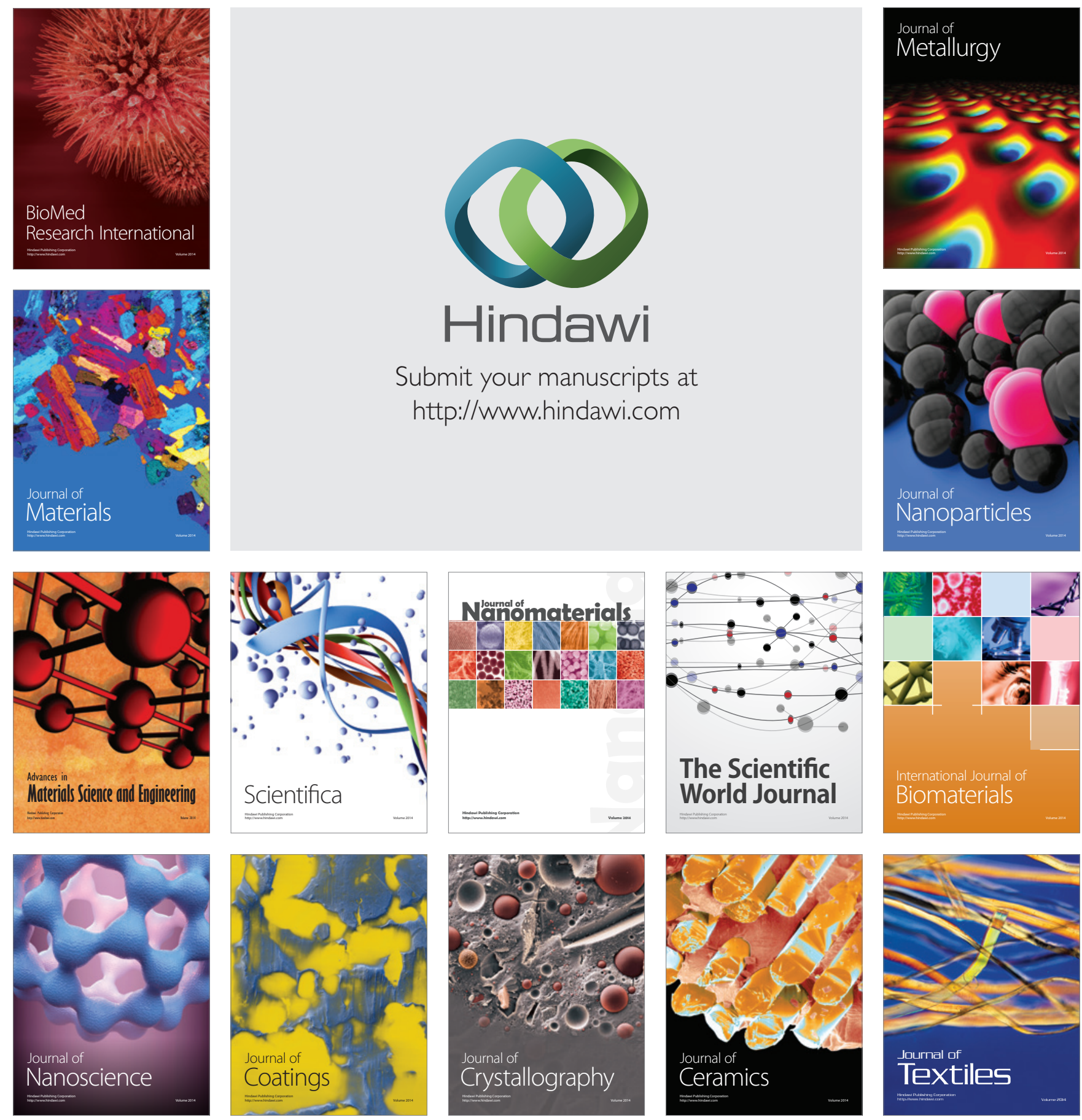